\title{
Lehmann rotation of cholesteric droplets driven by Marangoni convection ${ }^{\dagger}$
}

\begin{abstract}
P. Oswald ${ }^{1}$, J. Ignès-Mullol ${ }^{2,3}$ and A. Dequidt ${ }^{4}$
We show experimentally and theoretically that the Lehmann effect recently observed by Yoshioka and Araoka (Nature Com. 9, 432 (2018)) in emulsified cholesteric liquid crystal droplets under temperature gradients is due to Marangoni flows rather than to the thermomechanical or chemomechanical couplings often invoked to explain the phenomenon. Using colloidal tracers we visualize convection rolls surrounding stationary cholesteric droplets in vertical temperature gradients, while a shift in the position of internal point defects reveals the corresponding inner convection in nematic droplets thermomigrating in a horizontal temperature gradient. We attribute these phenomena to the temperature dependence of the surface tension at the interface between these partially-miscible liquids, and justify their absence in the usual case of purely lyophobic emulsions. We perform a theoretical analysis to help validate this hypothesis, demonstrating the strong dependence of the precession velocity on the configuration of the cholesteric director field.
\end{abstract}

\section{Introduction}

The rotation under a temperature gradient of cholesteric droplets was discovered by Lehmann in $1900^{1}$ and re-observed recently by several authors 2 . We recall that a cholesteric phase is a chiral nematic phase twisted in a single direction at equilibrium ${ }^{5}$. In all the recent experiments $2-4$, the cholesteric droplets coexist with their isotropic liquid at the transition temperature between the two phases. An alternative way of dispersing cholesteric droplets is emulsifying the cholesteric phase in an immiscible isotropic liquid, such as water or glycerol. This strategy, however, has failed to result in Lehmann rotation when the droplets are subjected to a temperature gradient. The situation has recently changed when experiments by Yoshioka and Araoka ${ }^{6}$ have shown rotation of cholesteric droplets dispersed in a partially-miscible isotropic liquid. Just as in the original Lehmann experiment, rotation occurs when a temperature gradient is applied, but it is much faster than in droplets coexisting with their isotropic phase, and it can be observed at all temperatures below the clearing transition. The possibility to achieve this thermomechanical rotation in an emulsion, rather than in the phase coexistence region is relevant, as it offers the required temperature versatility to employ such phe-

\footnotetext{
${ }^{1}$ Univ Lyon, ENS de Lyon, Univ Claude Bernard, CNRS, Laboratoire de Physique, F69342 Lyon, France

${ }^{2}$ Departament de Ciència de Materials i Química Física, Universitat de Barcelona, Martí i Franquès 1, 08028, Barcelona, Spain

${ }^{3}$ Institute of Nanoscience and Nanotechnology (IN2UB), Universitat de Barcelona, Barcelona, Spain

${ }^{4}$ Université Clermont Auvergne, CNRS, SIGMA Clermont, ICCF, F-63000 ClermontFerrand, France

$\dagger$ Electronic Supplementary Information (ESI) available: video of a growing particle assembly. See DOI: 10.1039/cXsm00000x/
}

nomenon in integrated lab-on-a-chip devices. It is, therefore, relevant to understand whether the rotation mechanism is the same in this experiment (which resembles that of Lehmann's when the latter dispersed the cholesteric phase in rosin or mineral oils) and the recent experiments performed in the coexistence region.

To answer this question, we recently analyzed the different mechanisms that could cause the Lehmann rotation of cholesteric droplets in either the coexistence region or in the emulsions ${ }^{7}$.

We immediately eliminated the explanation based on the existence of Leslie, Akopyan and Zel'dovich thermomechanical torques $\frac{8}{10}$. These torques exist $[1,15$ but are too small to explain the observed phenomena ${ }^{14}$. In addition they do not always predict the good sense of rotation of the droplets 14 . Another explanation suggested by a recent work by Bono et al 17 _ but not confirmed experimentally ${ }^{7}$ - lies on the existence of a flux of dissolved impurities (solute) across the droplets that would be responsible for a chemo-mechanical torque analog to the thermomechanical one. This torque, first predicted by de Gennes 18 , has already been observed in Langmuir monolayers 19 , 21 , freestanding films of Smectic $\mathrm{C}^{\star}$ liquid crystals ${ }^{22}$ and cholesteric phases 23 . The reason for eliminating this mechanism as the main explanation for the Lehmann effect observed in the coexistence region is that the rotation velocity of the droplets is independent of the chemical nature and the concentration of the impurities present in the samples. On the other hand, it is not excluded that this mechanism played a role in the experiment of Bono et al ${ }^{17}$ on the reversal of the sense of rotation of the droplets under strong UV illumination.

Another possibility is that the cholesteric material inside the droplets moves along the temperature gradient. Since the 
droplets are fixed in the temperature gradient, this mechanism implies that they should melt on their hot side and recrystallize on their opposite cold side. Such mechanism would indeed lead to the observation of rotating textures through the microscope on condition that the director field is twisted along the direction of observation. Such melting-recrystallization process, possible in the coexistence region between the cholesteric phase and its own isotropic liquid, becomes impossible when the droplets are dispersed in a liquid in which they are not, or poorly, soluble.

For this reason, we suggested in Ref. ${ }^{7}$ that another mechanism based on a Marangoni effect might be responsible for the rotation of the droplets when they are dispersed in a liquid different from the LC itself. In this paper, we show experimentally the existence of a strong Marangoni effect at the interface between the LC droplets and the dispersing isotropic liquid, and demonstrate that the associated flows are responsible for the observed rotation of the LC textures. Incidentally, we would like to emphasize here that this explanation was already mentioned by Lehmann himself as a footnote in his paper dating from 1 .

The structure of the paper is as follows. In Section 2 , we describe the experimental system. In Section 3 , we report our main experimental observations, including the textures of the droplets and their rotation velocity under a temperature gradient, and the evidence for the existence of convective rolls around the droplets, suggesting a Marangoni effect. Finally, in Section 4 we propose a model to interpret the rotation of the droplets. In the appendix, we include the exhaustive characterization of material parameters relevant for a quantitative analysis of the phenomenon.

\section{Experimental system}

The cholesteric phase was obtained by doping the liquid crystal 7CB (4-cyano-4'-n-heptylbiphenyl from Frinton Laboratories, USA) with $1.27 \% \mathrm{w} / \mathrm{w}$ of the chiral molecule R811 (R-(+)-octan2-yl 4-((4-(hexyloxy)benzoyl)oxy) benzoate from Merck, Germany). The cholesteric emulsion was obtained by dispersing 15 to $17 \% \mathrm{w} / \mathrm{w}$ of the cholesteric mixture in the fluorinated polyether polymer PolyFox-PF656 (PF, from Omnova Solutions, France). The chemical structure of PF is given in Ref. ${ }^{24}$. The emulsion was prepared by adding the required amount of the cholesteric mixture into PF, which is liquid in all the range of used temperatures, followed by mechanical shaking in a vortex stirrer until complete emulsification. For test experiments, we also dispersed the cholesteric liquid crystal in glycerol (Sigma-Aldrich, with a nominal purity of $99.7 \%$ ), which was used as received. For flow visualization, hydrophilic polystyrene tracer particles $1.7 \mu \mathrm{m}$ in diameter (Micromod micromer-M, PEG-COOH surface functionalization) were dispersed in PF. Briefly, $10-30 \mu 1$ of a 1:100 dilution of the stock particle solution was dried on a clean glass slide. $100 \mu 1$ of PF was added and the particles were redispersed by stirring with a pipette tip. A few minutes in an ultrasonic bath was used to properly disperse the particles prior to emulsification of the liquid crystal.

Using the above protocol, a large fraction of the cholesteric phase dissolves in $\mathrm{PF}$, in accordance with the phase diagram shown in Fig. A.1. The emulsion was introduced by capillarity in the gap between two parallel glass plates previously cleaned with sulfochromic acid. Nylon wires of diameter $70 \mu \mathrm{m}$ were used as a spacer. The vertical temperature gradient was imposed by sandwiching the sample between two ovens regulated at different temperatures. We refer to Ref. ${ }^{2}$ for a detailed description of the used setup. In the following, we shall note $\Delta T$ the temperature difference between the top and bottom ovens: $\Delta T=T_{u p}-T_{\text {down }}$. By assuming that the thermal conductivity of PF is close to that of the liquid crystal, we estimate the temperature gradient inside the sample to be $G(\mathrm{~K} / \mathrm{cm}) \approx 17 \Delta T$. In some experiments, we imposed a horizontal temperature gradient to our sample. In that case, the sample was drawn across two ovens separated by a small gap (for a description of the corresponding setup, see Ref. ${ }^{25}$ ). The temperature gradient inside the sample was locally measured with a thermocouple and reads $G(\mathrm{~K} / \mathrm{cm})=1.292 \Delta T$, where $\Delta T$ denotes here the temperature difference between the right and left ovens. All observations were performed by using a Laborlux 12POL microscope connected to a digital camera (Hamamatsu C4742-95) and a computer equipped with LabView for image acquisition.

\section{Results}

\subsection{Lehmann rotation of cholesteric droplets: basic obser- vations}

In agreement with the observations of Yoshioka and Araoka ${ }^{6}$, we found that the cholesteric droplets dispersed in a saturated solution of PF rotate much faster than droplets of similar sizes observed in coexistence with the isotropic liquid phase when they are submitted to a temperature gradient perpendicular to the glass plates.

Four main types of droplets were observed in our samples (Fig. 17). The first ones, by far the most frequent, are the spiraling droplets, in reference to their spiraling texture observed in natural light (Fig. 17). The second ones have a texture with a quadrupolar symmetry (Fig. 1 1 b) and will be called quadrupolar droplets for this reason. The third ones are the classical banded droplets (Fig. 11F) and the fourth ones are the concentric circles droplets (Fig.17), again in reference to their aspect in natural light. As the latter are axisymmetric, they do not rotate (more precisely, their texture does not rotate), contrary to the other types which rotate clockwise (anti-clockwise) when the temperature gradient points upwards (downwards). Note that the sense of rotation is the same here as in the classical Lehmann effect and corresponds to a positive Lehmann Rotatory Power (as defined in Ref. ${ }^{31}$ ). Each type of droplets exists in a well-defined range of radius $R$. More precisely, $10 \mu \mathrm{m}<R<20 \mu \mathrm{m}$ for the spiraling droplets, $8 \mu \mathrm{m}<R<11 \mu \mathrm{m}$ for the quadrupolar droplets, $11 \mu \mathrm{m}<R<15 \mu \mathrm{m}$ for the banded droplets and $R<10 \mu \mathrm{m}$ for the concentric circles droplets.

The same droplet can also display different textures over time. This is the case for the large spiraling droplets $(R>15 \mu \mathrm{m})$ that spontaneously change winding direction when the temperature gradient is reversed. This is visible in Fig. 2 showing the same droplet under a positive and negative temperature gradient. This indicates that the spiraling droplets of this size turn back on themselves when the temperature gradient is reversed. This process is shown in video S1 of the Supplementary Material. However, the same process is not observed for the spiraling droplets of small 

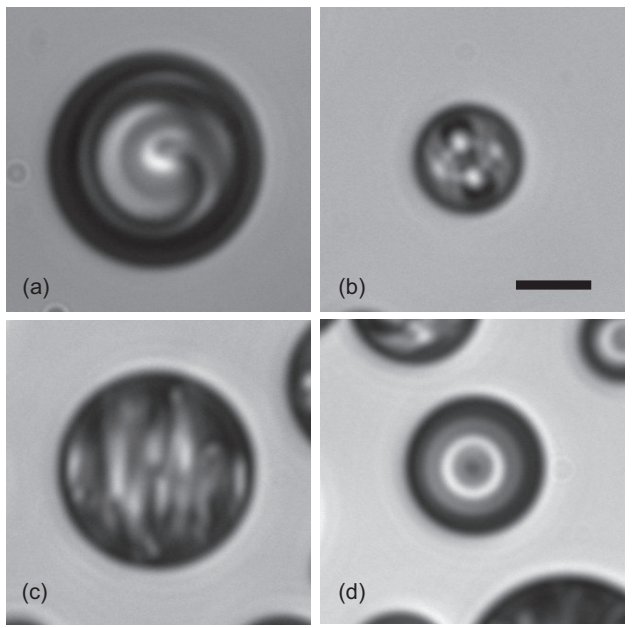

Fig. 1 The four main types of droplets observed in the sample $\left(T_{\text {ave }}=\right.$ $27.5^{\circ} \mathrm{C}, \Delta T=5^{\circ} \mathrm{C}$ ). a) Spiraling droplet; b) quadrupolar droplet; c) banded droplet; d) concentric circles droplets. The bar is $10 \mu \mathrm{m}$ long. All the photos were taken in natural light without polarizers.
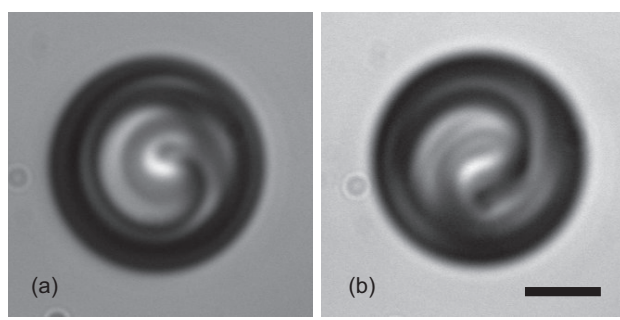

Fig. 2 A single spiraling droplet photographed when the temperature gradient points upwards (a): $\Delta T=5^{\circ} \mathrm{C}$ and downwards (b): $\Delta T=-5^{\circ} \mathrm{C}$. In these experiments, $T_{\text {ave }}=27.5^{\circ} \mathrm{C}$. The bar is $10 \mu \mathrm{m}$ long.

size $(R<15 \mu \mathrm{m})$. In that case, two behaviors may be observed depending on the value of the temperature gradient after it has changed sign. If the latter is not too large (typically $|\Delta T|<1.5^{\circ} \mathrm{C}$ ) the droplets remain unchanged and just rotate in the opposite direction. By contrast, they transform systematically into banded droplets if the temperature gradient is further increased (in absolute value) as can be seen in video S2 of the Supplementary Material. The reverse process, namely the transformation of a banded droplet into a spiraling one, is also systematically observed when the temperature gradient is reversed. This is the reason why it was impossible to observe the two senses of rotation with the same banded droplet (see curve (c) in Fig. 3). By contrast, the quadrupolar droplets and the concentric circle droplets remain unchanged when the temperature gradient is reversed. This is shown in video S3 of the Supplementary Material showing a quadrupolar droplet changing sense of rotation -but not texture- after a reversal of the temperature gradient.

We also systematically measured the rotation velocity of the different types of droplets as a function of the temperature gradient (Fig. 3), the average temperature of the sample (Fig. 4) and the radius of the droplets (Fig. 5). In all cases the rotation velocity is proportional to the temperature gradient and increases when the temperature increases (Fig. 3). The dependence on
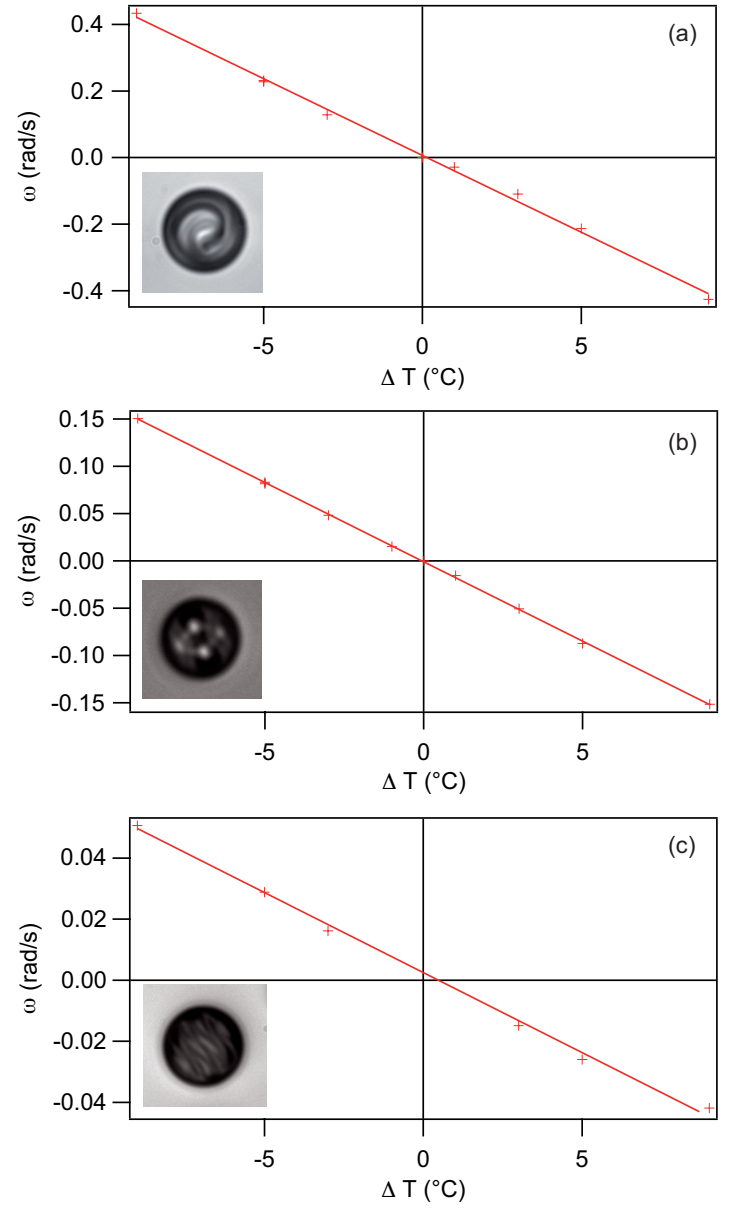

Fig. 3 Rotation velocity of the droplets at $T_{\text {ave }}=27.5^{\circ} \mathrm{C}$ as a function of the temperature difference between the top and bottom ovens. The curve (a) has been measured with the same spiraling droplet of radius $R=16.1 \mu \mathrm{m}$. In this example, the spiral has changed winding direction when the temperature gradient was reversed. The curve (b) has been measured with the same quadrupolar droplet of radius $R=9.9 \mu \mathrm{m}$. The curve (c) has been measured with two different banded droplets of radii $R=12.7 \mu \mathrm{m}(G>0)$ and $R=13.0 \mu \mathrm{m}(G<0)$. This could explain why the linear fit does not pass through the origin.

the average temperature is also the same for all the droplets whatever their type- as can be seen in Fig. 4(b). Finally, the rotation velocity of the banded droplets (respectively, of the spiraling droplets) decreases (respectively, increases) when their radius increases, while that of the quadrupolar droplet is independent of their radius within the experimental dispersion.

Last but not least, it must be noted that the texture of the spiraling droplets may change when the temperature gradient is increased. This effect is particularly visible on the spiraling droplets as can be seen in Fig. 6. There is also, for each type of droplet, a maximum temperature gradient above which droplets destabilize. An example is seen in video S4 of the Supplementary Material, showing the spiraling texture of a "large" droplet that destabilizes periodically in time. Note that a very similar behavior was described by Lehmann in his paper dating from 1900? We did not study the limit of stability of the droplets, nor their non-stationary regimes. By contrast, in this work we focussed our 

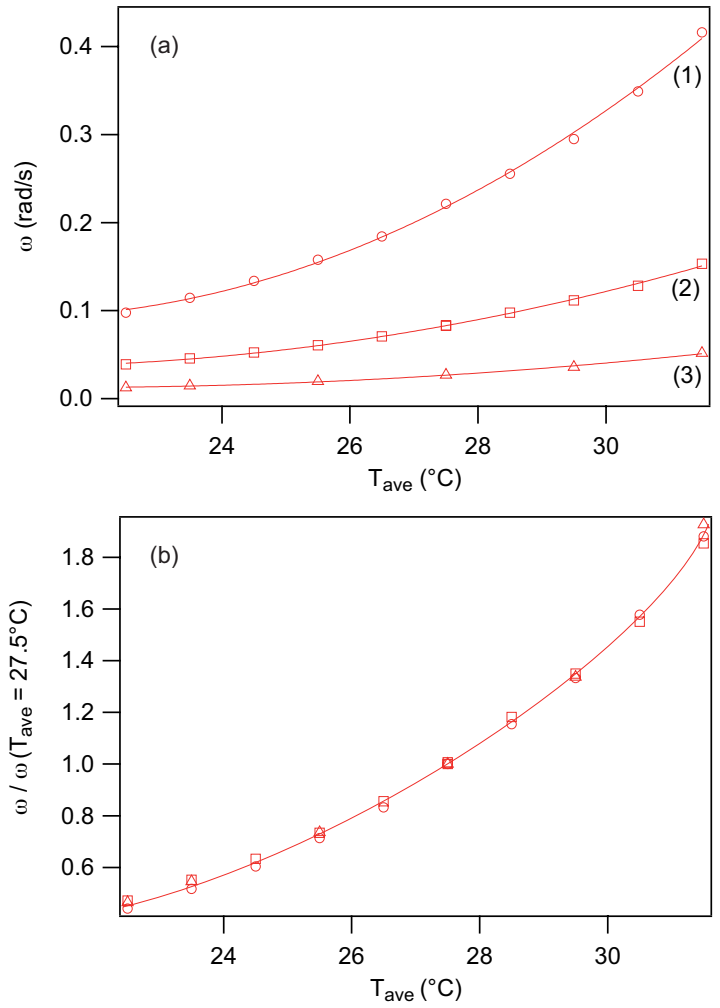

Fig. 4 (a) Rotation velocity as a function of the average temperature of the sample under a constant temperature difference $\Delta T=-5^{\circ} \mathrm{C}$. Each curve has been measured with the same droplet by increasing the temperature. Curve (1): spiraling droplet; curve (2) quadrupolar droplet; curve (3) banded droplet; (b) The same curves normalized with the rotation velocity measured at $T_{\text {ave }}=27.5^{\circ} \mathrm{C}$. The solid lines are just guides for the eye. During the experiments the radius of each droplet slightly decreased because of the slow dissolution of the cholesteric phase in PF. The initial (final) radii of the three droplets were 15 (13.4) $\mu \mathrm{m}$ (curve 1), 9.9 (8) $\mu \mathrm{m}$ (curve 2) and 14(11.9) $\mu \mathrm{m}$ (curve 3).

attention on the convective flows associated with the rotation of the droplets in the stationary regime.

\subsection{Experimental evidence of Marangoni convection. Verti- cal temperature gradients.}

To put into evidence convective flows, we dispersed spherical colloidal particles of diameter $1.5 \mu \mathrm{m}$ in the continuous phase. Fig. 7 shows the motion of one particle in the vicinity of a rotating spiraling droplet (see also video S5 of the Supplementary Material). From $t=0 \mathrm{~s}$ until $t=35 \mathrm{~s}$, the particle approaches the droplet while, after $t=45 \mathrm{~s}$, the particle departs from the droplet. During this motion the contrast of the particle changes, which means that the particle also moves in the vertical direction (the particle appears dark when approaching the observer and bright when departing from the observer). Fig. 8(a) shows how the distance of this particle to the droplet changes in time. From this graph the radial velocity of the particle was deduced (Fig. 8(b)). The periodic character of this motion shows that the particle has a convective motion in the vicinity of the droplet. This motion is still better visible in video S6 of the Supplementary Material showing a droplet surrounded by many particles. In that case, all

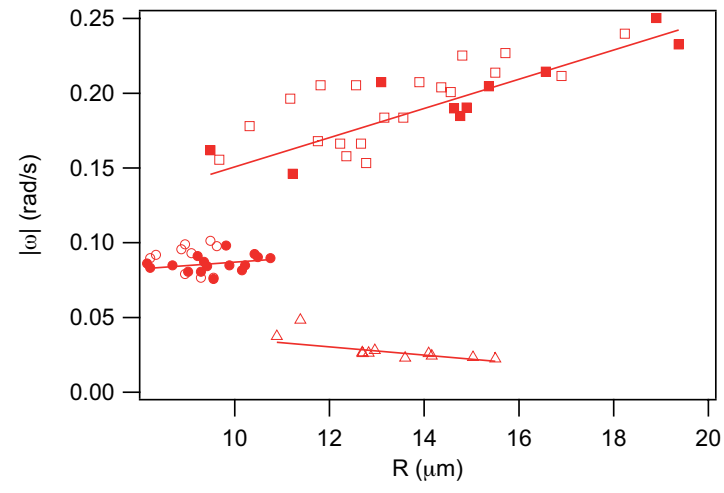

Fig. 5 Rotation velocity (in absolute value) measured at an average temperature of $27.5^{\circ} \mathrm{C}$ as a function of the radius of the droplets. Square: spiraling droplets; circles: quadrupolar droplets; triangles: banded droplets. The filled (open) symbols have been measured at $\Delta T=5^{\circ} \mathrm{C}\left(\Delta T=-5^{\circ} \mathrm{C}\right)$.
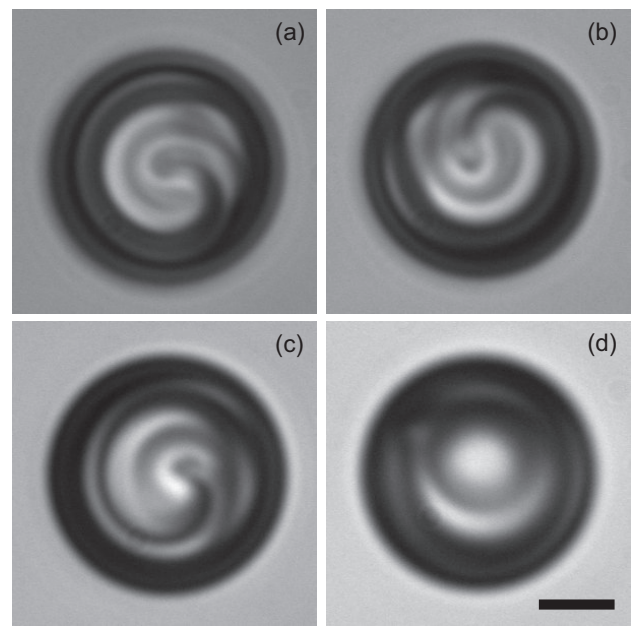

Fig. 6 The same spiraling droplet at four different temperature gradients: (a) $\Delta T=1^{\circ} \mathrm{C}$; (b) $\Delta T=3^{\circ} \mathrm{C}$; (c) $\Delta T=5^{\circ} \mathrm{C}$ and (c): $\Delta T=9^{\circ} \mathrm{C}$, for the same average temperature of $27.5^{\circ} \mathrm{C}$. At the larger temperature gradient, the contrast has changed significantly, meaning that the texture was distorted. The black bar is $10 \mu \mathrm{m}$ long.

the particles follow closed trajectories in vertical planes passing though the center of the droplets. Note that this experiment was performed with pure $7 \mathrm{CB}$, in the nematic phase, which explains why the droplet does not rotate. This observation confirms the existence of a strong convective motion across the sample thickness. From these data, we measured the period of revolution of the particles as a function of the major axis of their trajectory (namely half the horizontal distance they travel during a period of revolution). The results are collected in Fig. 9 and show clearly that the period of revolution increases when the major axis increases. The sense of rotation of the particles on their trajectories reverses when the temperature gradient changes sign as shown in Video S7 of the Supplementary Material, suggesting that these rolls are indeed due to a Marangoni effect at the surface of the droplets. As the velocity must be continuous at the surface of the droplets, similar convective rolls must also exist inside the droplets. However, these rolls are difficult to put into experimental evidence, since 


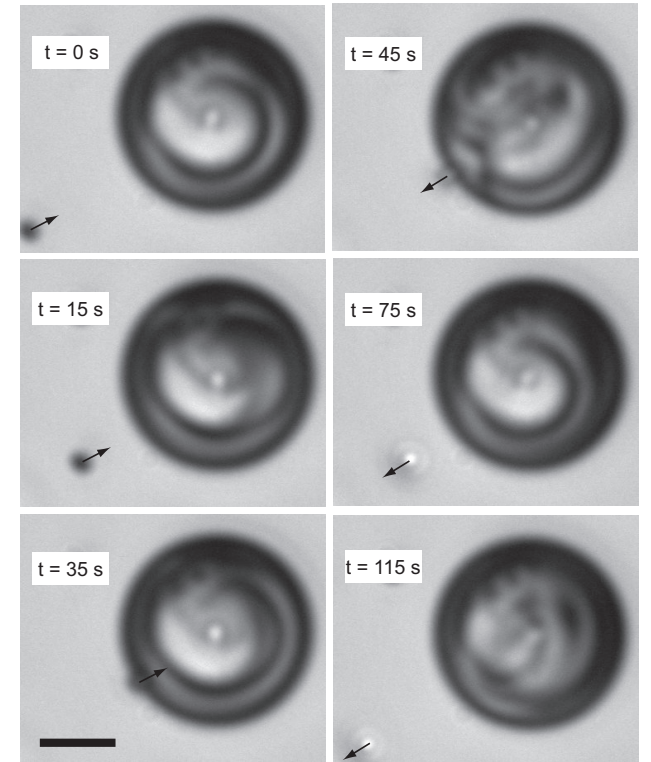

Fig. 7 Experimental evidence of the convective motion of a colloidal particle in the vicinity of a rotating spiraling droplet. The time is indicated on each micrograph. The arrows indicate the sense of displacement of the colloidal particle. The average temperature is $T_{\text {ave }}=32^{\circ} \mathrm{C}$ and $\Delta T=$ $5^{\circ} \mathrm{C}$. The black bar is $10 \mu \mathrm{m}$ long.

the colloidal particles are rejected from the cholesteric phase. Nevertheless there is no doubt that they exist and we propose that they are responsible for the observed Lehmann effect. This will be theoretically justified in Section 4. Before that, we looked for additional evidence of a strong Marangoni effect.

\subsection{Experimental evidence of Marangoni convection. Hori- zontal temperature gradients.}

It is well known that the Marangoni effect can cause the thermomigration of the droplets providing that the latter are free to migrate (for a review, see for instance Ref. ${ }^{32}$ ). This is the case if the temperature gradient is applied parallel to the glass plates. To check this expectation, we used an emulsion of pure 7CB (16 $\mathrm{wt} \%$ ) in PF. Note that, for simplicity, this experiment was done in the nematic phase. Fig. 10.a) shows a typical droplet photographed between crossed polarizers. The presence of a Maltese cross indicates that the director field is radial inside the droplet, an evidence that the anchoring is homeotropic at the interface with $\mathrm{PF}$ and the droplet contains a point defect of topological charge +1 at its center. This sample was then placed in the directional growth apparatus described in Ref. 25 , which imposes a constant temperature gradient parallel to the glass plates. We observed that all the droplets spontaneously migrate from the cold to the hot side of the sample. We systematically measured their migration velocity as a function of their radius for different temperature gradients $\left(\Delta T=5,10,20,30^{\circ} \mathrm{C}\right)$. All measurements were performed at $T_{\text {ave }}=30^{\circ} \mathrm{C}$. Fig. 11.(a) shows that the drift velocity is proportional to the temperature gradient and increases almost linearly with the droplet radius as long as the droplet diameter is less than the sample thickness. In addition, we observed that the
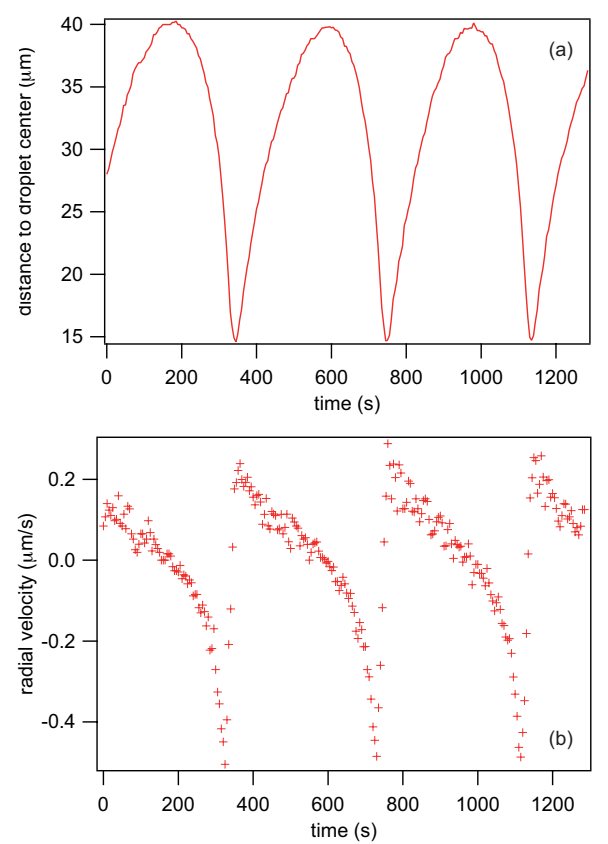

Fig. 8 (a) Distance between the particle and the droplet center as a function of time; (b) Corresponding radial velocity as a function of time.

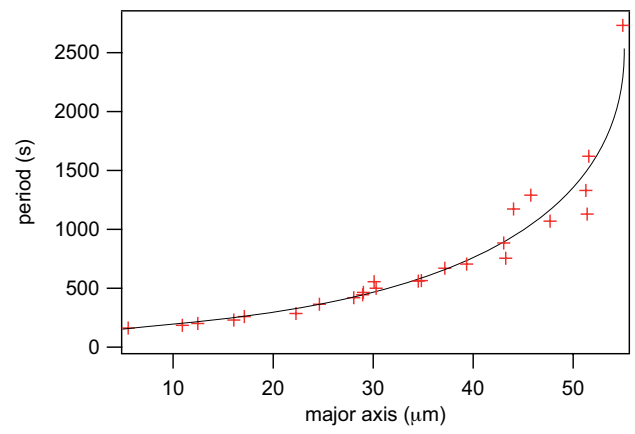

Fig. 9 Period of rotation of the colloidal particles as a function of the major axis of their trajectory. The solid line is just a guide for the eye.

central point defect shifts from the center of the droplet towards the hot side by a distance $\delta x$ (Fig. $10(\mathrm{~b})$ ). This shift, which increases with the radius of the droplet and is proportional to the temperature gradient (see Fig. 12), is clearly due to a flow inside the droplet, schematically represented by the dashed lines in Fig. 10(b). Note that a similar phenomenon was already observed in previous experiments on active emulsion of nematic droplets obtained by dispersing the liquid crystal in a surfactant solution 26. 28. Obviously, the convection flows we have put into evidence in this horizontal oven configuration will also be present for the vertical arrangement. They will actually be of similar amplitude, since the applied vertical temperature gradients are of the same order of magnitude as their horizontal counterparts.

All these observations on the thermomigration of the droplets are compatible with a strong Marangoni effect and suggest that the surface tension between PF and 7CB, $\gamma$, changes significantly with temperature, which we have confirmed experimentally (Sec: 

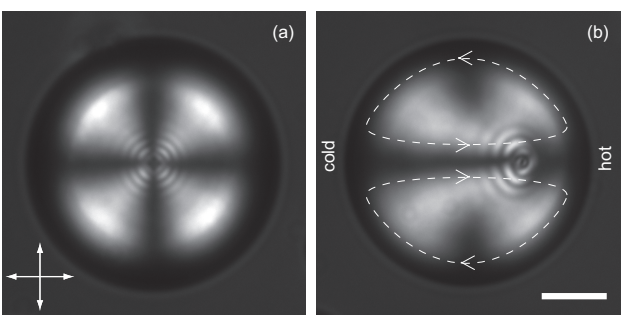

Fig. 10 Nematic droplet observed between crossed polarizers. (a) Droplet at rest $(\Delta T=0)$; (b) Droplet moving from the left to the right under the action of a horizontal temperature gradient $\left(\Delta T=20^{\circ} \mathrm{C}\right)$. The right shift of the central defect reveals the existence of a flow inside the droplet represented by the two dashed lines. The white bar is $20 \mu \mathrm{m}$ long.
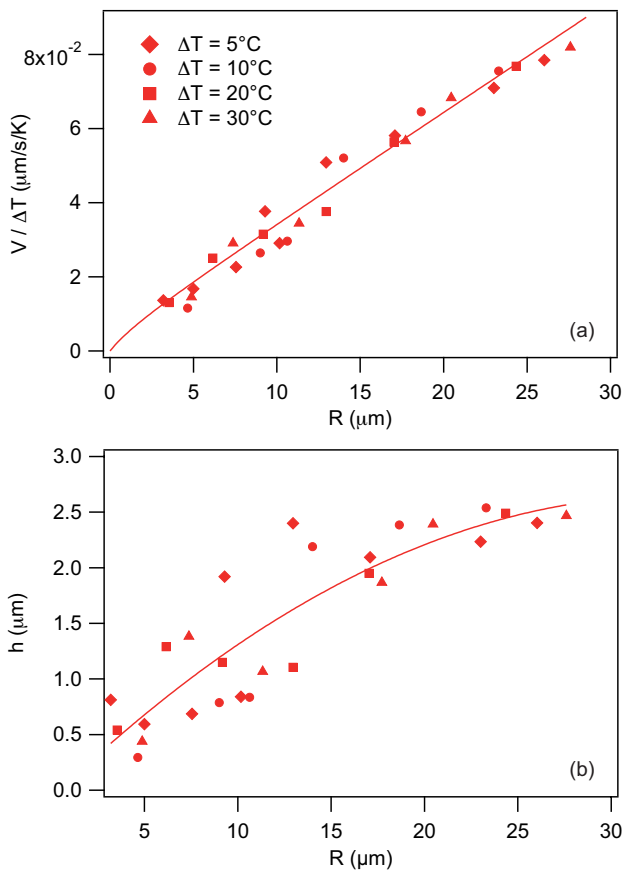

Fig. 11 (a) Drift velocity of the droplet normalized to the temperature gradient as a function of the radius. The measurements were performed at $T=30^{\circ} \mathrm{C}$ for different temperature gradients. (b) Thickness of the lubrication layer calculated from Eq. (18) and the values of $\gamma^{\prime}$ and $v$ measured experimentally at $T=30^{\circ} \mathrm{C}$ (see Figs. A.4 and A.5. The solid line is a parabolic fit of the data. The solid line in graph (a) has been calculated from this fit and Eq. (18).

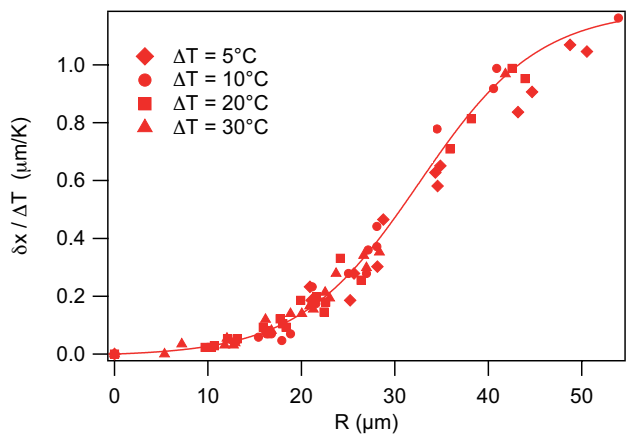

Fig. 12 Normalized shift of the defect as a function of the droplet radius. The solid line is just a guide for the eye.
A.5). We find that $\gamma$ strongly decreases with temperature, with a coefficient $\gamma^{\prime}=\frac{d \gamma}{d T} \simeq-0.08 \mathrm{dyn} / \mathrm{cm} / \mathrm{K}$ in the used temperature range (Fig. A.6(b)). These measurements allow to compare our measured velocities with those predicted for the Marangoni thermomigration of a droplet in an infinite medium 35 ,

$$
V_{\infty}=\frac{3 G R \gamma^{\prime}}{2 \mu+3 \mu^{\prime}} \frac{\kappa}{2 \kappa+\kappa^{\prime}}
$$

where $G$ is the temperature gradient far from the droplet, $\mu\left(\mu^{\prime}\right)$ is the viscosity of PF (7CB) and $\kappa\left(\kappa^{\prime}\right)$ is the thermal conductivity of $\mathrm{PF}$ (7CB). In our experiments the droplets are confined between two glass plates so that Eq. 1] is not directly applicable. For this situation, approximate expressions were proposed for confined droplets moving at large distances (with respect to their radius) from the surface of the glass plates 36 . This is not the case in our experiments where 7CB droplets move in the close vicinity of the upper plate because of the buoyancy force. For such a situation, a semi-empirical formula for the droplet velocity $V$ was proposed 37 in the form

$$
V=V_{\infty} \frac{1}{1+\frac{R}{4 \pi h}},
$$

where $h$ is the typical thickness of the lubrication layer that forms between the droplet and the glass plate. We used this formula to fit our data by assuming that the thermal conductivities of the two liquids were the same. Doing this, we calculated from the data of Fig. 11(a) and Eq. (2) the thickness $h$ of the lubrication layer. The results are shown in Fig. 11(b). As expected, the typical thickness $h$ of the lubrication layer lies in the $\mu$ m-range. An interesting point is that this thickness increases when the radius -and consequently the velocity- of the droplets increase. More precisely $h$ is of the order of $R / 10$ which means that the measured velocity $V$ is typically two times smaller that the velocity calculated in an infinite medium because of the friction on the glass plate.

\section{Modeling the Lehmann effect driven by Marangoni convection}

\subsection{General expression for the rotation velocity}

In this section, we assume that the internal texture of the droplet rotates at constant velocity and remains unchanged during the rotation (stationary regime). In this case, the elastic energy of the droplet remains constant. As a result,

$$
\frac{\mathrm{d}}{\mathrm{d} t} \iiint_{\mathrm{drop}} f \mathrm{~d}^{3} \vec{r}=0
$$

by denoting by $f$ the elastic Frank energy density $\sqrt[5]{ }$. This gives

$$
\iiint_{\text {drop }} \vec{h}_{\perp} \cdot \frac{\partial \vec{n}}{\partial t} \mathrm{~d}^{3} \vec{r}=0
$$

where $\vec{h}_{\perp}$ is the component orthogonal to the director $\vec{n}$ of the molecular field $\vec{h}=-\frac{\delta f}{\delta \vec{n}}$. Note that the volume integrals are calculated over the whole droplet.

The torque equation reads (by omitting the thermomechanical 
terms of Leslie, Akopyan and Zel'dovich)

$$
\gamma_{1} \frac{\partial \vec{n}}{\partial t}=\vec{h}_{\perp}-\gamma_{2} \underline{A} \vec{n}-\gamma_{1}(\vec{v} \cdot \vec{\nabla}) \vec{n}+\gamma_{1} \vec{\Omega} \times \vec{n}
$$

which gives

$$
\begin{gathered}
\gamma_{1} \iiint_{\text {drop }}\left(\frac{\partial \vec{n}}{\partial t}\right)^{2} \mathrm{~d} V= \\
\iiint_{\text {drop }}\left(-\gamma_{2} \underline{A} \vec{n}-\gamma_{1}(\vec{v} \cdot \vec{\nabla}) \vec{n}+\gamma_{1} \vec{\Omega} \times \vec{n}\right) \cdot \frac{\partial \vec{n}}{\partial t} \mathrm{~d}^{3} \vec{r} .
\end{gathered}
$$

In these equations, $\vec{\Omega}=\frac{1}{2} \overrightarrow{\operatorname{Curl}} \vec{v}$ is the local rotation rate, $\underline{A}$ is the symmetric strain rate tensor of component $A_{i j}=\frac{1}{2}\left(v_{i, j}+v_{j, i}\right)$ and $\gamma_{1} \equiv \alpha_{3}-\alpha_{2}$ and $\gamma_{2} \equiv \alpha_{3}+\alpha_{2}$ are two viscosities of the cholesteric phase (with $\alpha_{2}$ and $\alpha_{3}$ two of the five Leslie viscosity coefficients ${ }^{5}$ ).

Because the texture rotates as a rigid body, one must have $\mathrm{e}^{2}$

$$
\frac{\partial \vec{n}}{\partial t}=\omega \vec{e}_{z} \times \vec{n}-\omega \frac{\partial \vec{n}}{\partial \theta}
$$

where $\vec{e}_{z}$ is the unit vector along the $z$-axis and $\theta$ the polar angle in cylindrical coordinates $(r, \theta, z)$. By replacing $\frac{\partial \vec{n}}{\partial t}$ by its expression (7) in Eq. (6) and by setting $\frac{\delta \vec{n}}{\delta \theta}=\frac{\partial \vec{n}}{\partial \theta}-\vec{e}_{z} \times \vec{n}$, we obtain:

$$
\omega=\frac{\iiint_{\operatorname{drop}}\left(\gamma_{2} \underline{A} \vec{n}+\gamma_{1}[(\vec{v} \cdot \vec{\nabla}) \vec{n}-\vec{\Omega} \times \vec{n}]\right) \cdot \frac{\delta \vec{n}}{\delta \theta} \mathrm{d}^{3} \vec{r}}{\iiint_{\operatorname{drop}} \gamma_{1}\left(\frac{\delta \vec{n}}{\delta \theta}\right)^{2} \mathrm{~d}^{3} \vec{r}} .
$$

This expression can be simplified if the velocity field (and, consequently $\underline{A}$ ) does not depend on $\theta$, which we will assume in the following. In this case, the integral

$$
I_{2}=\iiint_{\text {drop }} \underline{A} \vec{n} \cdot \frac{\delta \vec{n}}{\delta \theta} \mathrm{d}^{3} \vec{r}
$$

vanishes for symmetry reasons (see Appendix B) and $\omega$ takes the simplified form:

$$
\omega=\frac{\iiint_{\operatorname{drop}}[(\vec{v} \cdot \vec{\nabla}) \vec{n}-\vec{\Omega} \times \vec{n}] \cdot \frac{\delta \vec{n}}{\delta \theta} \mathrm{d}^{3} \vec{r}}{\iiint_{\operatorname{drop}}\left(\frac{\delta \vec{n}}{\delta \theta}\right)^{2} \mathrm{~d}^{3} \vec{r}},
$$

where $\gamma_{1}$ and $\gamma_{2}$ do no longer appear explicitly.

This equation establishes a direct relationship between the angular velocity of the texture and the director and velocity fields. Of course this analysis does not fully resolve the problem because the director and velocity fields are unknown and must be determined by solving simultaneously the torque, momentum and heat equations. This is a formidable task that can be done only numerically. However, Eq. 10 can be useful to estimate the rotation velocity provided adequate director and velocity fields are given. This is what we propose to do in the next two sections by choosing two simple ansatzes for the textures.

\subsection{Application to a simple helix texture}

Here we assume that the droplet texture consists of a simple helix making an angle $\alpha$ with the vertical $z$ axis. This is the simplest cholesteric texture we can imagine. Although this texture does not satisfy the homeotropic anchoring conditions, it allows
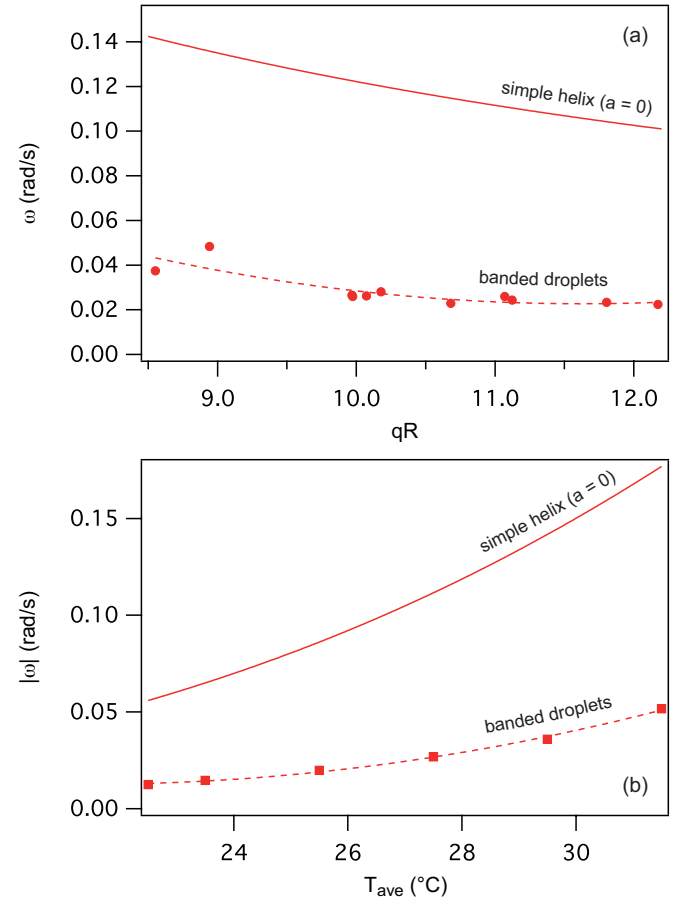

Fig. 13 Comparison between the calculated velocity for a horizontal simple helix and the measured velocity for the banded droplets. (a) Rotation velocity as a function of the dimensionless product $q R\left(\Delta T=5^{\circ} \mathrm{C}\right.$ and $T_{\text {ave }}=27.5^{\circ} \mathrm{C}$ ). (b) Rotation velocity as a function of the average temperature $\left(R=14 \mu \mathrm{m}\right.$ and $\left.\Delta T=5^{\circ} \mathrm{C}\right)$.

to check that, indeed, coupling of a chiral texture with Marangoni flows can lead to texture rotation.

The director field for a simple helix can be written in the form

$$
\vec{n}=\cos (q u+\psi) \vec{e}_{v}+\sin (q u+\psi) \vec{e}_{w}
$$

where $\psi$ is an arbitrary phase, $u=\left(r \vec{e}_{r}+z \vec{e}_{z}\right) \cdot \vec{e}_{u}$ is the coordinate along the helical axis parallel to the unit vector $\vec{e}_{u}=$ $\cos (\alpha) \vec{e}_{z}+\sin (\alpha) \vec{e}_{x}, \vec{e}_{w}=\vec{e}_{y}$ and $\vec{e}_{v}=\vec{e}_{w} \times \vec{e}_{u}$. For the velocity field, we suppose that the flow inside the droplet is not very different from the one calculated in an infinite medium and we assume that the cholesteric phase behaves as an isotropic liquid of viscosity $\mu^{\prime} \approx \alpha_{4} \underline{5}$. In this limit the flow corresponds to the so-called Hill vortex and reads, in polar coordinates $(r, \theta, z)^{35}$ :

$$
\left\{\begin{array}{l}
v_{r}=-\beta r z \\
v_{\theta}=0 \\
v_{z}=\beta\left(z^{2}+2 r^{2}-R^{2}\right),
\end{array}\right.
$$

where $\beta=\frac{3 G \gamma^{\prime}}{\left(2 \mu+3 \mu^{\prime}\right) R} \frac{\kappa}{2 \kappa+\kappa^{\prime}}$. Note that $\vec{v}$ is independent of $\theta$ so that Eq. (8) can be directly used. With this director field and this velocity field the integrals in Eq. (8) can be calculated analytically (we do it using Mathematica). This calculation gives:

$$
\omega=\frac{A \beta R \sin ^{2} \alpha}{1+B \sin ^{2} \alpha}
$$


with

$$
\left\{\begin{array}{l}
A=\frac{32 q^{5} R^{5}+15 \cos (2 \psi)\left[6 q R \cos (2 q R)+\left(-3+4 q^{2} R^{2}\right) \sin (2 q R)\right]}{64 q^{4} R^{4}} \\
B=\frac{q^{2} R^{2}}{5}-\frac{1}{2}-\frac{3 \cos (2 \psi)(-2 q R \cos (2 q R)+\sin (2 q R))}{16 q^{3} R^{3}}
\end{array}\right.
$$

In our experiments $4<q R<16$. In this range of $q R$ the terms in $\psi$ in the expressions of $A$ and $B$ are completely negligible. This leads to the simple formula:

$$
\omega=\frac{5 q R \sin ^{2} \alpha}{10+\left(2 q^{2} R^{2}-5\right) \sin ^{2} \alpha} \frac{G \gamma^{\prime}}{2 \mu+3 \mu^{\prime}}
$$

by taking $\kappa=\kappa^{\prime}$. This formula shows that $\omega$ is proportional to $G$ and is odd in $q$ as observed experimentally. It predicts the good sense of rotation since $\omega$ and $G$ are of opposite signs when $q>0$ and $\gamma^{\prime}<0$. The calculated velocity also changes as a function of $R$ and $T_{\text {ave }}$ as the experimental velocity does (see Fig. 13). However, the calculated velocity is typically three times larger than the measured velocity for the banded droplets. Several factors can explain this quantitative discrepancy between the theory and the experiment. One of them is that the experimental flow is likely to be different from the one used in the calculation, since a Hill vortex is only expected for an infinite medium and with isotropic liquids. In particular, our droplets are in contact with a glass plate, which should reduce the velocity by at least a factor of 2 if one refers to the reduction of velocity observed in the experiment on the thermomigration of the droplets (see the discussion at the end of Sec 3.3. Another factor, perhaps more crucial, is that the texture considered in this calculation does not satisfy the homeotropic boundary conditions at the surface of the droplet. This could lead to profound changes in both the texture and the associated rotation velocity. This point is analyzed in the next paragraph.

\subsection{Application to a texture with a single point defect}

In order to account for the homeotropic anchoring at the surface of the droplet, we propose another ansatz for the director field, which is a combination of the director field of a simple helix and the one of a radial hedgehog defect:

$$
\vec{n}=\frac{f(\rho) \vec{n}_{r}+[1-f(\rho)] \vec{n}_{h}}{\left\|f(\rho) \vec{n}_{r}+[1-f(\rho)] \vec{n}_{h}\right\|}
$$

with

$$
\left\{\begin{array}{l}
\vec{n}_{h}=\cos (q u) \vec{e}_{v}+\sin (q u) \vec{e}_{w} \quad \text { (helix) } \\
\vec{n}_{r}=\frac{r \vec{e}_{r}+z \vec{e}_{z}}{\sqrt{r^{2}+z^{2}}} \quad \text { (radial hedgehog) }
\end{array}\right.
$$

The switch function $f(\rho)$ (where $\rho=\sqrt{r^{2}+z^{2}}$ is the distance from the center of the drop) varies from 0 to 1 when $\rho$ varies from 0 to $R$. It will be taken in the form $f(\rho)=\frac{\rho}{R} e^{k\left(\frac{\rho}{R}-1\right)}$, where $k$ is a positive number to determine.

This texture satisfies by construction the homeotropic anchoring on the surface of the droplet and contains a point defect of charge +1 which is situated on the $\vec{e}_{v}$ axis at a distance $\rho_{d}$ from

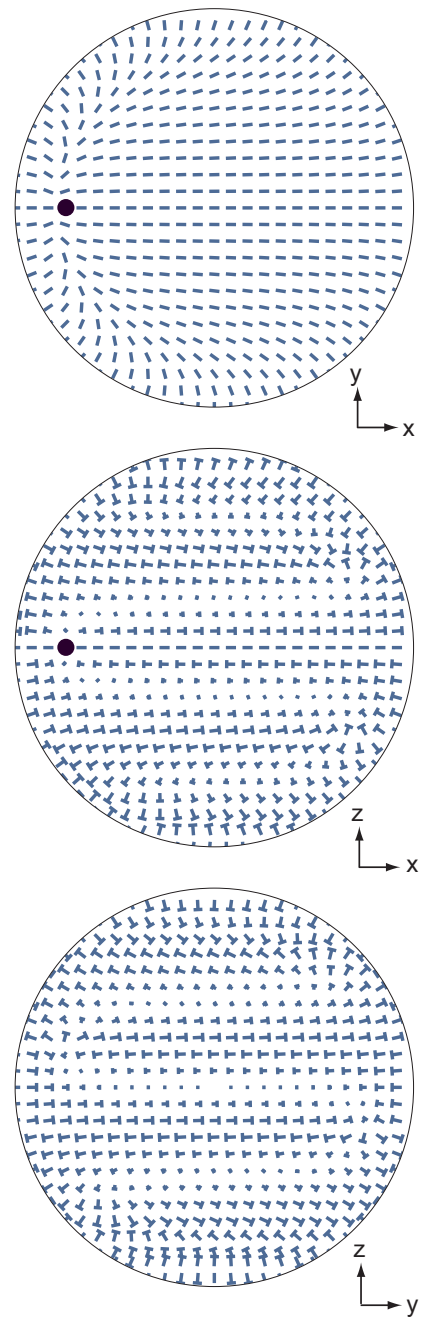

Fig. 14 Director field corresponding to the ansatz proposed in Sec. 4.2 in three orthogonal planes passing through the center of the droplet when $\alpha=0$ (see text). The black dot marks the position of the point defect of charge +1 on the $x$ axis. The director field was calculated by taking $q R=7$ and the value of $k$ that minimizes the elastic energy (here $k=2.306$ ). The classical "nail" representation of the director field was used.

the center given by $f\left(\rho_{d}\right)=1 / 2$ (a similar texture was already proposed in Refs. $\frac{3839}{39}$ ). On the point defect $\vec{n}$ is not defined $(\vec{n}=\overrightarrow{0} / 0)$. The axes $\vec{e}_{u}, \vec{e}_{v}$ and $\vec{e}_{w}$ are defined as in the previous paragraph. Close to the center of the droplet the director field is shaped like an helix with its helical axis in the $(x, z)$ plane inclined by an angle $\alpha$ with respect to the $z$ axis. The corresponding director field is shown in Fig. 14 when $\alpha=0$ and $q R=7$. The parameter $k$ was determined by minimizing with Mathematica the total elastic energy of the droplet. The calculation was simplified by assuming isotropic elasticity $\left(K=K_{1}=K_{2}=K_{3}\right)$. The result is shown in Fig. 15 where we also show how the distance of the point defect to the drop surface changes with $q R$. This graph shows that there is a critical value of $q R$ of the order of 4.6 below which $k$ vanishes and $\rho_{d} \rightarrow 0.5$. This value of $q R$ certainly marks the passage towards another type of texture (quadrupolar droplets or concentric circle droplets). In this case the description 


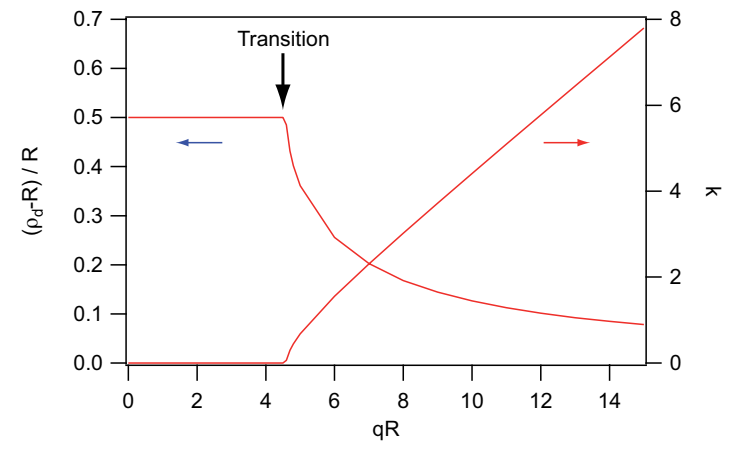

Fig. 15 (a) Parameter $k$ which minimizes the elastic energy and dimensionless distance between the defect and the surface of the droplet $\left(\rho_{d}-R\right) / R$ as a function of $q R$. The value $q R=4.5$ marks a transition towards another texture.

given in Eq. 17 is no longer pertinent.

We then calculated the rotation velocity when the helical axis in the center of the droplet is parallel $(\alpha=0)$ and perpendicular $(\alpha=\pi / 2)$ to the temperature gradient. The calculations were performed numerically with Mathematica by using Eq. 10 and the velocity field given in Eq.13. In order to compare with the previous calculation, we plotted the dimensionless velocity $\omega / \beta R$ as a function of $q R$ in Fig. 16 for the two orientations of the helix and the two types of droplets. As we can see the results for the two ansatzes are very different, in particular when the helical axis is parallel to the temperature gradient. Indeed the calculation gives $\omega=0$ for a simple helix and a large rotation velocity when a point defect is introduced to satisfy the homeotropic anchoring at the surface of the droplet. This shows that the rotation velocity crucially depends on the texture. It must be emphasized that the rotation velocity found for the texture with the point defect is typically 5 to 8 times larger when the helix is vertical than when it is horizontal. For comparison, we reported in Fig. 16 the experimental points measured for the banded droplets (triangles) and for the spiraling droplets (squares) (see Fig. 5). To account for the friction on the glass plate, $\beta$ was divided by a factor of 2 according to the remark made in the previous paragraph. As we can see the rotation velocities predicted by our simplified model are compatible in order of magnitude with the velocities measured experimentally.

\section{Conclusions}

We have shown the existence of Marangoni flows around cholesteric droplets emulsified in the fluorinated solvent PolyFoxPF656, when they are submitted to a temperature gradient. We have also demonstrated that these flows are due to the temperature dependence of the surface tension between the liquid crystal and PF. Marangoni flows are put into experimental evidence with the thermomigration of the droplets when the temperature gradient is parallel to the glass plates limiting the sample. When the gradient is applied perpendicularly to the glass plates, droplets can no longer migrate but their inner textures rotate because of the coupling between the director field and the flow. This rotation, which is only observed in the cholesteric phase and for

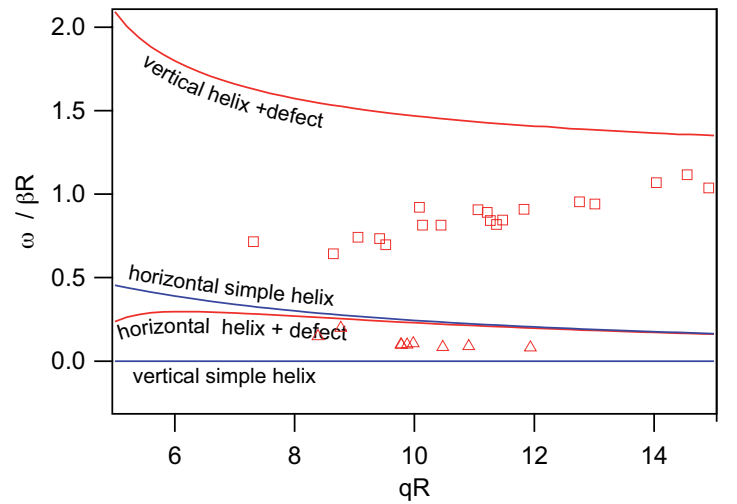

Fig. 16 Dimensionless rotation velocity $\omega / \beta R$ as a function of $q R$. The blue curves have been calculated with a simple helix. The red curves have been calculated by introducing a point defect of charge +1 to satisfy the homeotropic anchoring at the surface of the droplet. The presence of a point defect radically changes the rotation velocity when the helix is vertical. For comparison, we have reported on this graph the experimental points corresponding to the banded (triangles) and spiraling (squares) droplets (same data as in Fig. 5.

a chiral director field, is similar to the classical Lehmann effect observed in the coexistence region between the cholesteric phase and its isotropic liquid. We have verified that neither Lehmann rotation nor Marangoni convection is observed when the cholesteric droplets are dispersed in glycerol, where the temperature variation of the interfacial tension is very low (Fig. A.7). We, therefore, conclude that Lehmann rotation in emulsified cholesteric droplets is inseparable from the existence of Marangoni flows. We have also found that the speed of rotation of the textures in the cholesteric droplets is often much faster that the speed of rotation of the convection rolls. This is particularly true for the spiraling droplets. However we did not detect any systematic flow in the plane of the sample, in agreement with the observations of Yoshioka and Araoka ${ }^{6}$. This puts into evidence that, similarly to our own observations of the usual Lehmann effect ${ }^{15 / 40}$, here there is no rigid body rotation of the suspended droplets.

In order to support our conclusions, we have formulated a general relationship between the rotation velocity, the director field (or the texture) and the velocity field inside the droplet. With this analysis, we have estimated the rotation velocity of different textures and have found a good qualitative agreement with experiments. In the future, it would be important to better determine the internal texture of the droplets by analyzing their microscopy images. This is a difficult task because of the strong deviations of the light rays across the droplets. The next step, once the textures have been characterized, would be to numerically solve the equations of nematodynamics in the temperature gradient for each texture. Finally, we emphasize that the mechanism proposed here explains both the Lehmann rotation recently reported by Yohioka and Araoka of cholesteric droplets dispersed in $\mathrm{PF}^{6}$ and also the rotation of cholesteric droplets observed in the experiments of Yamamoto and Sano during their self-propulsion in an aqueous surfactant solution 28 . 


\section{Acknowledgements}

P. O. warmly thanks M. Costa-Gomes and T. Moufawad for their help in measuring the density of the PF, E. Freyssingeas for his help in measuring the viscosity of the pure PF with the Haake rheometer, F. Bunel for his technical help during the construction of the experimental device used to measure the surface tension, and G. Poy for suggesting the director field used in Eq. 17 Dr W. Becker from Merck and Dr S. Dumas from OMNOVA Solutions are also warmly thanked for providing free samples of R811 and PF. J. I.-M. thanks Ecole Normale Supérieure de Lyon for hospitality, J. Ortín for his help in measuring the viscosity with a TA Instruments rheometer, and acknowledges funding from MINECO (project FIS2016-78507-C2-1-P, AEI/FEDER, EU).

\section{A Physical properties of the used materials}

\section{A.1 Phase diagram of the mixture of PolyFox-PF656 and 7CB}

The phase diagram of the mixture between PF and 7CB is shown in Fig. A.1. In practice, 10 mixtures with different concentrations were prepared and homogenized at high temperature (typically $90^{\circ} \mathrm{C}$ in order that the $7 \mathrm{CB}$ dissolves completely in the PF). Each mixture was then introduced by capillarity between two parallel glass plates separated by $70 \mu \mathrm{m}$ spacers. The filling was done in an oven heated to a temperature of $90^{\circ} \mathrm{C}$ in order that the $\mathrm{PF}$ and the $7 \mathrm{CB}$ do not separate. The samples were then quickly transferred into a Mettler hot stage FP82. The phase transition temperatures were detected under the microscope by observing the samples between crossed polarizers. A particular care was taken to ensure that the samples equilibrated each time the temperature was changed (the kinetics of nucleation and growth of the droplets are very slow). This was very important, in particular to precisely measure the upper temperature at which the droplets disappear. The reader will also note that the transition line between the $\mathrm{N}+\mathrm{I}$ and $\mathrm{I}+\mathrm{I}$ zones is not horizontal, as it should be for a true binary mixture. This is due to the fact that the PF is not pure but polydisperse with respect to molecular weight. This also explains why we observe the coexistence of three phases (Nematic and Isotropic droplets coexisting with an Isotropic liquid) in a narrow temperature band of about $0.2^{\circ} \mathrm{C}$ around this limit. This band is too narrow to be shown in the phase diagram. Note also that the PF dissolves very little in the 7CB as we already noted in Ref. ${ }^{7}$. In practice the concentration of PF inside the 7CB droplets is always less than $0.5 \%$ by weight.

\section{A.2 Pitch of the cholesteric mixture}

The pitch of the cholesteric mixture of $7 \mathrm{CB}+1.27 \% \mathrm{w} / \mathrm{w}$ of R811, saturated in PF, was measured by first separating the cholesteric phase emulsified in PF by centrifugation once material equilibrium had been reached in the emulsion. A Cano wedge method was used ${ }^{5}$. The pitch -close to $8 \mu \mathrm{m}$ - changes little with temperature as shown in Fig. A.2 This value is larger than that found for the cholesteric phase before saturation with PF (about $6 \mu \mathrm{m}$ ). This is likely due to a difference in the miscibility of 7CB and R811 in PF.

\section{A.3 Density measurements}

The density of pure $\mathrm{PF}$ and $\mathrm{PF}$ with $10 \mathrm{wt} \%$ 7CB was measured using a commercial density meter using a U-shaped vibrating-tube (model DMA 5000M from Anton Paar). The calibration of the equipment was verified with air and distilled water before and after each measurement. The estimated uncertainties of this equipment are $0.0001 \mathrm{~g} / \mathrm{cm}^{3}$ and $0.01 \mathrm{~K}$ for density and temperature, respectively. The density curves of the pure $\mathrm{PF}$ and of the mixture $\mathrm{PF}+10 \mathrm{wt} \% 7 \mathrm{CB}$ are shown in Fig. A.3. From these two curves, we extrapolated the density of the $\mathrm{PF}$ saturated in $7 \mathrm{CB}$ as a function of temperature (solid line in Fig A.3). Note that a linear extrapolation was used by taking for each temperature the value of the concentration at saturation given by the phase diagram of Fig. A.1.

\section{A.4 Viscosity measurements}

The viscosity of pure PF and PF with 10wt\% 7C was measured with different rheometers. We successively measured with a home-made piezoelectric rheometer (for a description see Refs. .2930 ) the viscosity of the pure PF and that of the mixture $\mathrm{PF}+10 \mathrm{wt} \% 7 \mathrm{CB}$ and we then extrapolated these results to find the viscosity of PF saturated in 7CB. The corresponding curves are shown in Fig. A.4(a). Note that the viscosity we have found for the pure $\mathrm{PF}$ is $30 \%$ smaller than that given by Omnova Solutions in their documentation. For this reason, we measured again the viscosity of the pure PF with two different commercial rotating rheometers (Haake and TA Instruments DHR-2). The results obtained with these two rheometers are in good agreement with our measurements as shown in Fig. A.4(a). Finally, we measured the viscosity of the cholesteric phase with our piezoelectric rheometer (Fig. A.4(b)). In this experiment, the cholesteric phase was not aligned, so this viscosity represents a typical average viscosity.

\section{A.5 Surface tension measurements}

For the surface tension measurement, a droplet of 7CB was injected at the end of a hollow stainless steel tube in a reservoir (a VWR disposable cuvette in optical polystyrene of dimension 10x10x45 mm) filled with PF saturated in 7CB. The tube was linked to a syringe filled with 7CB. The internal (external) diameter of the tube was $0.8(1.2) \mathrm{mm}$. The reservoir was placed inside

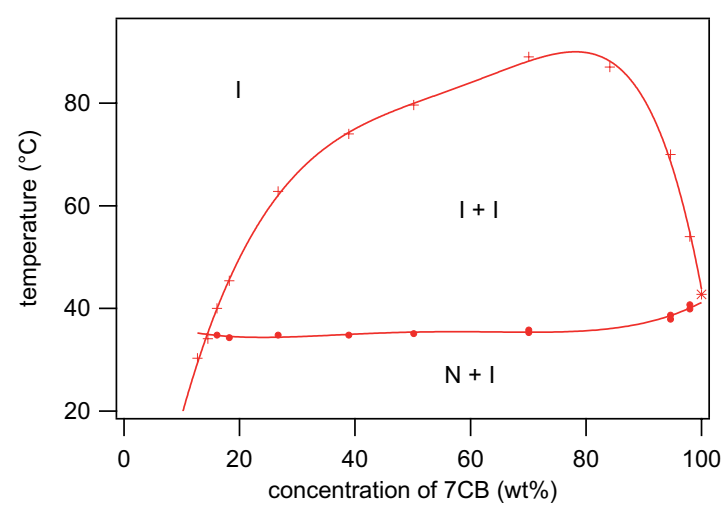

Fig. A.1 Phase diagram of the mixture 7CB + PF PF656. 


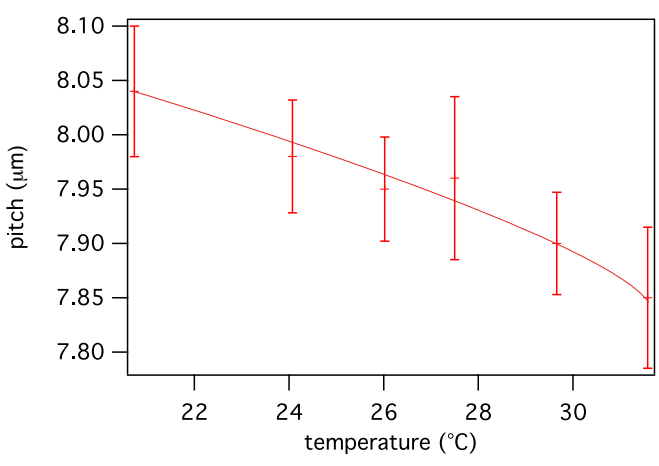

Fig. A.2 Pitch of the cholesteric phase once dispersed in the PF PF656. The solid line is just a guide for the eye.

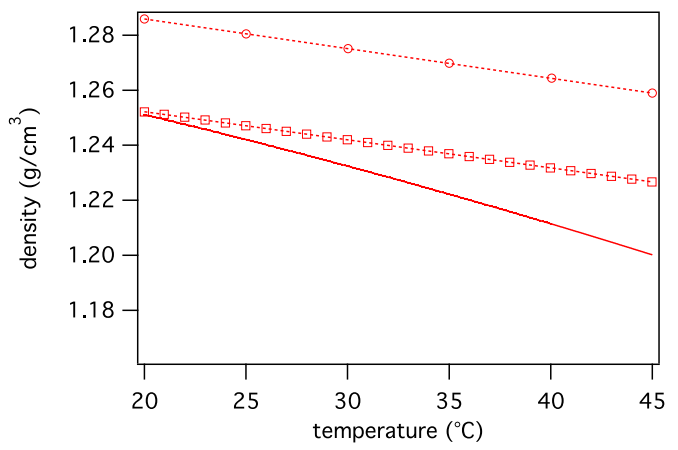

Fig. A.3 Density of the pure PF (circles) and of the isotropic mixture $\mathrm{PF}+10 \mathrm{wt} \% 7 \mathrm{CB}$ (squares) as a function of temperature. The solid line shows the density of the PF saturated in 7CB obtained from a linear extrapolation of the previous data.

an oven regulated in temperature to within $\pm 0.05^{\circ} \mathrm{C}$. The temperature was measured with a thermocouple that dips into the PF and was placed at less than $1 \mathrm{~mm}$ from the drop. The drop was visualized through a macroscope Leica M420 horizontally fixed on an optical table. Because of the difference of density between the two liquids, the drop elongates and shifts from a spherical shape when its size becomes comparable to the gravitational capillary length. An example is shown in Fig. A.5. The surface tension was deduced from an analysis of the shape of the drop at equilibrium. In practice two lengths were measured: the equatorial diameter $d_{e}$ and the diameter $d_{s}$ at a distance $d_{e}$ down from the top of the droplet. From this measurement, the shape-dependent quantity $S=d_{s} / d_{e}$ was calculated. The surface tension was obtained from the formula:

$$
\gamma=\frac{\Delta \rho g d_{e}^{2}}{H}
$$

where $g$ is the gravity constant, $\Delta \rho=\rho_{\mathrm{PF}}-\rho_{7 \mathrm{CB}}$ is the density difference and $1 / H$ is a quantity that has been precisely tabulated versus the measured quantity $S$ (see for instance Table II-7 in Ref. ${ }^{33}$ ). For $\rho_{\mathrm{PF}}$ we used the values extrapolated from our measurements (solid line in Fig. A.3) and for $\rho_{7 \text { Св }}$ we used the values given in Ref. $\frac{34}{3}$. The surface tension obtained in this way is shown in Fig. A.6(a). From a 7th order polynomial fit of this curve, we deduced the temperature coefficient of the surface tension $\gamma^{\prime}=\frac{d \gamma}{d T}$
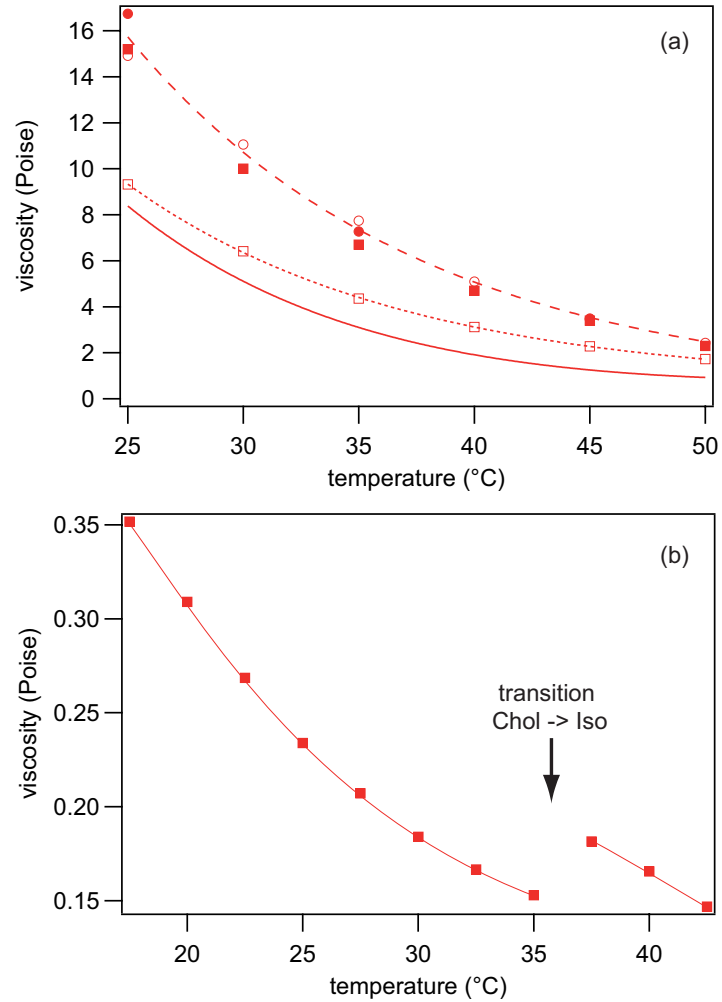

Fig. A.4 (a)Viscosity of the pure PF (circles) and of the isotropic mixture $\mathrm{PF}+10 \mathrm{wt} \% 7 \mathrm{CB}$ (squares) as a function of temperature. The open circles have been measured with the piezoelectric rheometer and the filled circles and squares with the two rotating rheometers. The dashed line is a global exponential fit of all the data obtained with the pure PF. The dotted line is an exponential fit of the data for the mixture PF $+10 w t \% 7 C B$. The solid line is a linear extrapolation from the two previous fits of the viscosity of the PF saturated in 7CB. (b) Average viscosity of the cholesteric phase saturated in PF measured with the piezoelectric rheometer as a function of temperature.

(Fig. A.6(b)).

We performed similar measurements with glycerol as continuous phase. Our results are shown in Fig. A.7. In this case we used the density values of the glycerol/water mixtures given in the website ${ }^{41}$ by taken a fraction of glycerol by volume of $99.7 \%$. We also took care that the glycerol did not hydrate during the experiment by sealing the reservoir with a Teflon stopper.

\section{B Proof of the vanishing of the integral in $\gamma_{2}$ when the strain rate tensor is indepen- dent of $\theta$}

To show that $I_{2}=0$, we must first note that $\frac{\delta \vec{n}}{\delta \theta}=\frac{\partial n_{r}}{\partial \theta} \vec{e}_{r}+\frac{\partial n_{\theta}}{\partial \theta} \vec{e}_{\theta}+$ $\frac{\partial n_{z}}{\partial \theta} \vec{e}_{z} \equiv \frac{\partial \vec{n}}{\partial \theta}$ in cylindrical coordinates. By integrating $I_{2}$ by parts in cylindrical coordinates and by using the fact that $\theta$ is periodic, we obtain

$$
I_{2}=-\iiint_{\text {drop }} \frac{\partial(\underline{A} \vec{n})}{\partial \theta} \cdot \vec{n} \mathrm{~d}^{3} \vec{r} .
$$

Note that the possible presence of point or line defects in the droplet does not change the result because the boundary term $(\underline{A} \vec{n}) \cdot \vec{n}$ is bounded and integrated over an area of vanishing mea- 

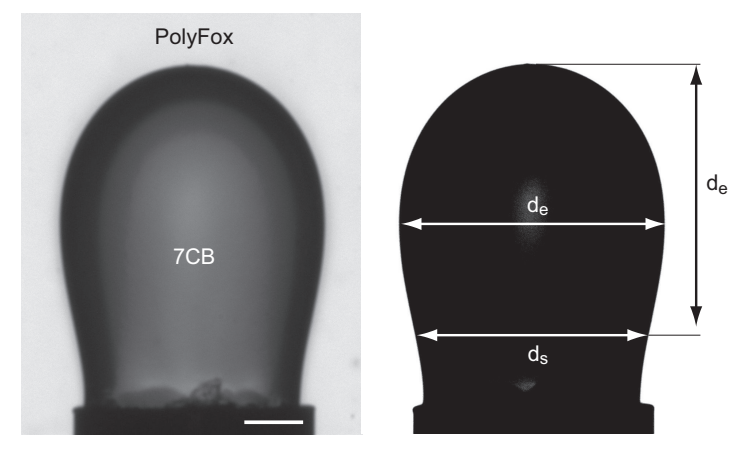

Fig. A.5 Drop of 7CB immersed in a bath of PF saturated in 7CB. On the right image, the contrast has been increased with Image $\mathrm{J}$ to measure the two lengths $d_{e}$ and $d_{s}$. The white bar is $250 \mu \mathrm{m}$ long. $T=30^{\circ} \mathrm{C}$.

sure around the defect.

As $\underline{A}$ does not depend on $\theta$,

$$
I_{2}=-\iiint_{\mathrm{drop}} \frac{A}{\partial \theta} \cdot \vec{n} \overrightarrow{\mathrm{d}^{3}} \vec{r} .
$$

Knowing that $\underline{A}=\underline{A}^{T}$ by definition, it comes

$$
I_{2}=-\iiint_{\operatorname{drop}} \underline{A} \vec{n} \cdot \frac{\delta \vec{n}}{\delta \theta} \mathrm{d}^{3} \vec{r}=-I_{2},
$$

so that $I_{2}=0$.

\section{References}

1 O. Lehmann, Structur, System und magnetisches Verhalten flüssiger Krystalle und deren Mischbarkeit mit festen, Ann. Phys. (Leipzig) 307, 649-705 (1900).

2 P. Oswald, A. Dequidt, Measurement of the Continuous Lehmann Rotation of Cholesteric Droplets Subjected to a Temperature Gradient, Phys. Rev. Lett. 100, 217802 (2008).

3 T. Yamamoto, M. Kuroda and M. Sano, Three-dimensional analysis of thermomechanically rotating cholesteric liquid crystal droplets under a temperature gradient, Europhys. Lett. 109, 46001 (2015).

4 F. Ito, J. Yoshioka and Y. Tabe, Heat-Driven Rotation in Cholesteric Droplets with a Double Twisted Structure, J. Phys. Soc. Jpn 85, 114601 (2016).

5 P. Oswald, P. Pieranski Nematic and Cholesteric Liquid Crystals: Concepts and Physical Properties Illustrated by Experiments (Taylor \& Francis, CRC press, Boca Raton, 2005).

6 J. Yoshioka, F. Araoka, Topology-dependent self-structure mediation and efficient energy conversion in heat-flux-driven rotors of cholesteric droplets, Nature Com. 9, 432 (2018).

7 P. Oswald, G. Poy, Role of impurities in the Lehmann effect of cholesteric liquid crystals: Towards an alternative model, Phys. Rev. E 98, 032704 (2018).

8 F. M. Leslie, Some Thermal Effects in Cholesteric Liquid Crystals, Proc. Roy. Soc. A 307, 359-372 (1968).

9 R. S. Akopyan and B. Y. Zel'dovich, Thermomechanical effects in deformed nematics, JETP 87, 1660-1669 (1984).

10 G. Poy and P. Oswald, On the existence of the thermomechan-
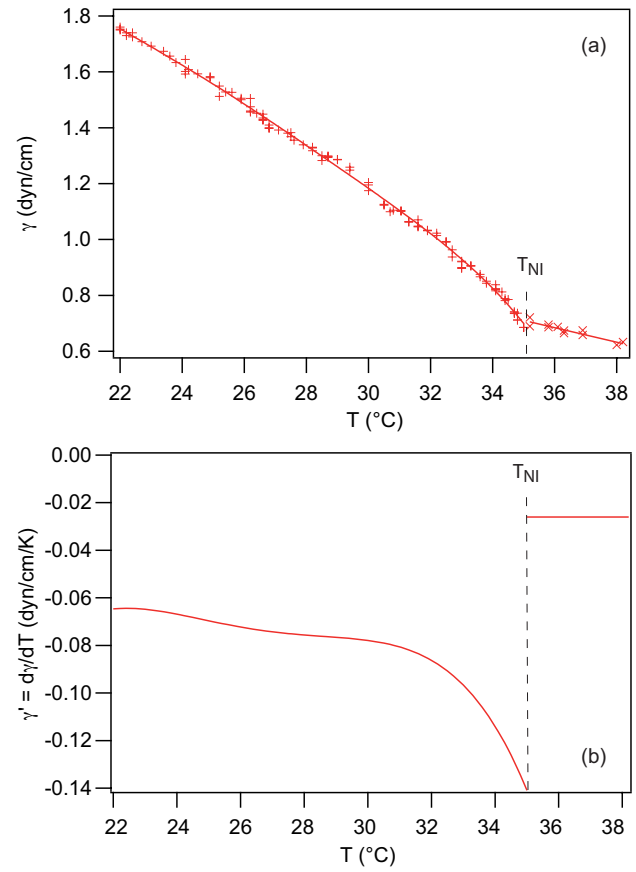

Fig. A.6 (a) Surface tension of the 7CB-PF interface as a function of temperature. The solid lines are polynomial and linear fits of the data in the nematic and isotropic phases, respectively; (b) Temperature parameter of the surface tension in the nematic phase as a function of temperature calculated from the polynomial fit of the surface tension.

ical terms of Akopyan and Zel'dovich in cholesteric liquid crystals, Liq. Cryst. 45, 1428-1442 (2018).

11 N. Éber, I. Jánossy, An experiment on the thermomechanical coupling in cholesterics, Mol. Cryst. Liq. Cryst. Lett. 72, 233-238 (1982).

12 A. Dequidt, A. Żywociński, P. Oswald, Lehmann effect in a compensated cholesteric liquid crystal: Experimental evidence with fixed and gliding boundary conditions, Eur. Phys. J. E 25, 277-289 (2008).

13 P. Oswald, A. Dequidt, Direct measurement of the thermomechanical Lehmann coefficient in a compensated cholesteric liquid crystal, Europhys. Lett. 83, 16005 (2008).

14 P. Oswald, Leslie thermomechanical power in diluted cholesteric liquid crystals, Europhys. Lett. 108, 36001 (2014).

15 P. Oswald, G. Poy, A. Dequidt, Lehmann rotation of twisted bipolar cholesteric droplets: role of Leslie, Akopyan and Zel'dovich thermomechanical coupling terms of nematodynamics, Liq. Cryst. 44, 969-988 (2016).

16 P. Oswald, Microscopic vs macroscopic origin of the Lehmann effect in cholesteric liquid crystals, Eur. Phys. J. E 35, 10 (2012).

17 S. Bono, S. Sato, Y. Tabe, Unidirectional rotation of cholesteric droplets driven by UV-light irradiation, Soft Matter 13, 65696575 (2017).

18 P. G. de Gennes The Physics of Liquid Crystals (Clarendon Press, Oxford, 1974).

19 Y. Tabe, H. Yokoyama, Coherent collective precession of molecular rotors with chiral propellers, Nat. Mater. 2, 806-809 (2003). 


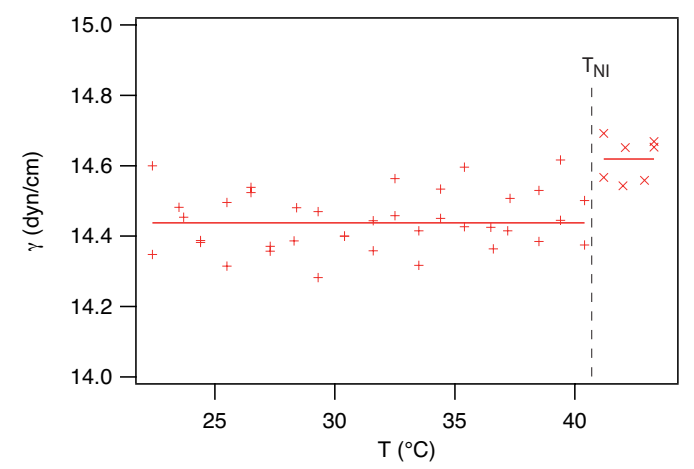

Fig. A.7 Surface tension of the 7CB-glycerol interface as a function of temperature. The surface tension is constant within our experimental errors. Note that the surface tension is about 10 times larger than that measured with PF whereas $\gamma^{\prime}$ is at least 10 times smaller. We attribute these differences to the difference in solubility of $7 C B$ in these two liquids.

20 P. Milczarczyk-Piwowarczyk, A. Żywociński, K. Noworyta, R. Holyst, Collective rotations of ferroelectric liquid crystals at the air/water interface, Langmuir 24, 12354-12363 (2008).

21 F. Bunel, J. Ignés-Mullol, F. Sagués, P. Oswald, Chemical Leslie effect in Langmuir monolayers: a complete experimental characterization, Soft Matter 14, 4835-4845 (2018).

22 K. Seki, K. Ueda, Y.-I. Okumura, Y. Tabe, Nonequilibrium dynamics of 2D liquid crystals driven by transmembrane gas flow, J. Phys.: Condens. Matter 23, 284114 (2011).

23 A. Darmon, M. Benzaquen, S. Cǒpar, O. Dauchot, T. LopezLeon, Topological defects in cholesteric liquid crystal shells, Soft Matter 12, 9280 (2016).

24 A. Kozbial, W. Guan, L. Li, Manipulating the molecular conformation of a nanometer-thick environmentally friendly coating to control the surface energy, J. Mater. Chem. A 5, 9752-9759 (2017).

25 P. Oswald, M. Moulin, P. Metz, J.C. Géminard, P. Sotta, L. Sallen, An improved directional growth apparatus for liquid crystals: applications to thermotropic and lyotropic systems, J. Phys. III 3, 1891-1907 (1993).

26 S. Herminghaus, C. C. Maass, C. Krüger, S. Thutupalli, L. Goehring, C. Bahr, Interfacial mechanisms in active emulsions, Soft Matter 10, 7008-7022 (2014).

27 C. Krüger, G. Klös, C. Bahr, C. C. Maass, , Phys. Rev. Lett. 117, 048003 (2016).

28 T. Yamamoto, M. Sano, Chirality-induced helical selfpropulsion of cholesteric liquid crystal droplets, Soft Matter 13, 3328-3333 (2017).

29 P. Oswald, D. Le Fur, Réalisation d'une cellule de déformation adaptée à l'étude des cristaux liquides smectiques, C. R. Acad. Sci. Paris 297, 699-702 (1983).

30 P. Oswald, Viscoelasticity of a homeotropic nematic slab , Phys. Rev. E 92, 062508 (2015).

31 P. Oswald, L. Jørgensen, A. Żywociński, Lehmann rotatory power: a new concept in cholesteric liquid crystals, Liq. Cryst. 38, 601-613 (2011).

32 A. Karbalaei, R. Kumar, H. J. Cho, Thermocapillarity in Mi- crofluidics - A Review, Micromachines 7, 13 (2016).

33 A. W. Adamson Physical Chemistry of Surfaces (Wiley, New York, 1990).

34 T. Shirakawa, T. Hayakawa, T. Tokuda, Pressure-VolumeTemperature Relations and Isotropic-Nematic Transitions for a 4'-n-Alkyl-4-cyanobiphenyl Homologous Series, J. Chem. Phys. 87, 1406-1408 (1983).

35 N. O. Young, J. S. Goldstein, M. J. Block, The motion of bubbles in a vertical temperature gradient, J. Fluid Mech. 6, 350356 (1959).

36 S. H. Chen, Movement of a Fluid Sphere in the Vicinity of a Flat Plane with Constant Temperature Gradient, J. Coll. Inter. Sci. 230, 157-170 (2000).

37 Yu. K. Bratukhin, A. L. Zuev, Thermocapillary drift of an air bubble in a horizontal Hele-Shaw cell, Fluid Dynamics 19, 393 398 (1984).

38 D. Seč, T. Porenta, M. Ravnik, S. Žumer, Geometric frustration of chiral ordering in cholesteric droplets, Soft Matter 8, 1198211988 (2012).

39 G. Posnjak, S. Čopar, I. Muševic, Hidden topological constellation and polyvalent charges in chiral nematic droplets, Nature Com. 8, 14594 (2017).

40 G. Poy, P. Oswald, Do Lehmann cholesteric droplets subjected to a temperature gradient rotate as rigid bodies?, Soft Matter 12, 2604 (2016).

41 http://www.met.reading.ac.uk/ sws $04 \mathrm{cdw} /$ viscosity_calc.html 